\title{
Agôn
}

Revue des arts de la scène

$5 \mid 2012$

L'entrée en scène

\section{Les états d'esprit de l'entrée en scène}

Entretien réalisé par Julie Sermon

\section{Gaëtan Vourc'h et Julie Sermon}

\section{CpenEdition}

Journals

Édition électronique

URL : http://journals.openedition.org/agon/2350

DOI : 10.4000 /agon.2350

ISSN : 1961-8581

Éditeur

Association Agôn

Référence électronique

Gaëtan Vourc'h et Julie Sermon, «Les états d'esprit de l'entrée en scène », Agôn [En ligne], 5 | 2012, mis en ligne le 14 janvier 2012, consulté le 15 septembre 2020. URL : http://journals.openedition.org/ agon/2350

Ce document a été généré automatiquement le 15 septembre 2020.

Association Agôn et les auteurs des articles 


\title{
Les états d'esprit de l'entrée en scène
}

\author{
Entretien réalisé par Julie Sermon
}

Gaëtan Vourc'h et Julie Sermon

\section{NOTE DE L'ÉDITEUR}

Entretien réalisé en juillet 2012

Formé à l'Ecole du Passage (Niels Arestrup) puis à l'ENSATT (Théâtre : Nada Strancar, Alain Knapp. Cinéma : Jean-Pierre Améris), Gaëtan Vourc'h a aussi étudié le théâtre à l'Université de Glasgow (Ecosse). Acteur, il travaille entre autres avec Noëlle Renaude, Florence Giorgetti, Edith Scob, Christophe Huysman, Irène Bonnaud, Maurice Benichou, Damien Caille-Perret, Philippe Minyana, Agnès Bourgeois, Frédéric Maragnani. Depuis 2002, il a par ailleurs participé à tous les projets du Vivarium Studio-Philippe Quesne, dont L'Effet de Serge, pour lequel il a reçu un Obie Award pour les représentations à New York en 2010.

Julie sermon: Comment, en tant qu'acteur, te prépares-tu à l'entrée en scène? Que représente pour toi ce passage de seuil?

Gaëtan VOURC'H : La question de l'entrée en scène arrive assez tard, finalement : elle ne se pose qu'à partir du moment où les spectateurs sont dans la salle. Durant les répétitions, les questions que je me pose sont plutôt celles tournées vers la fiction: comment j'amorce la situation, qu'est-ce que cette situation peut raconter et quoi faire avec.

J.S. : Une fois qu'arrivent les représentations, à quoi penses-tu, sur quoi te concentres-tu au moment où tu dois faire ton entrée devant les spectateurs?

G.V.: Comment dire... C'est un peu comme monter un escalier : il faut partir du bon pied. Cela peut être le pied gauche ou bien le pied droit, peu importe. Mais il faut que ce soit le bon. En tout cas, il faut en être convaincu parce que si on hésite on se prend la marche ! La différence entre les répétitions et les représentations, c'est que 
l'entrée en scène devient le moment de prise de contact avec le public : face à cette grosse énergie qui se dégage des spectateurs, j'arrive avec une énergie toute petite. Je ne peux pas espérer fasciner les gens d'entrée de jeu. Il faut que je me concentre sur ce que j'ai à faire là. Et il faut que je reste présent à ce que je fais. Ce qui n'est pas gagné parce c'est un moment où l'esprit carbure (ma mère est dans la salle, j'ai oublié de mettre dans ma poche le faux sang, etc.). Pourtant, si je parviens à cette présence, je m'installe dans une sorte de confiance qui va ensuite me porter, scène après scène, jusqu'à la fin du spectacle.

\section{Gaëtan Vourc'h en Linda}

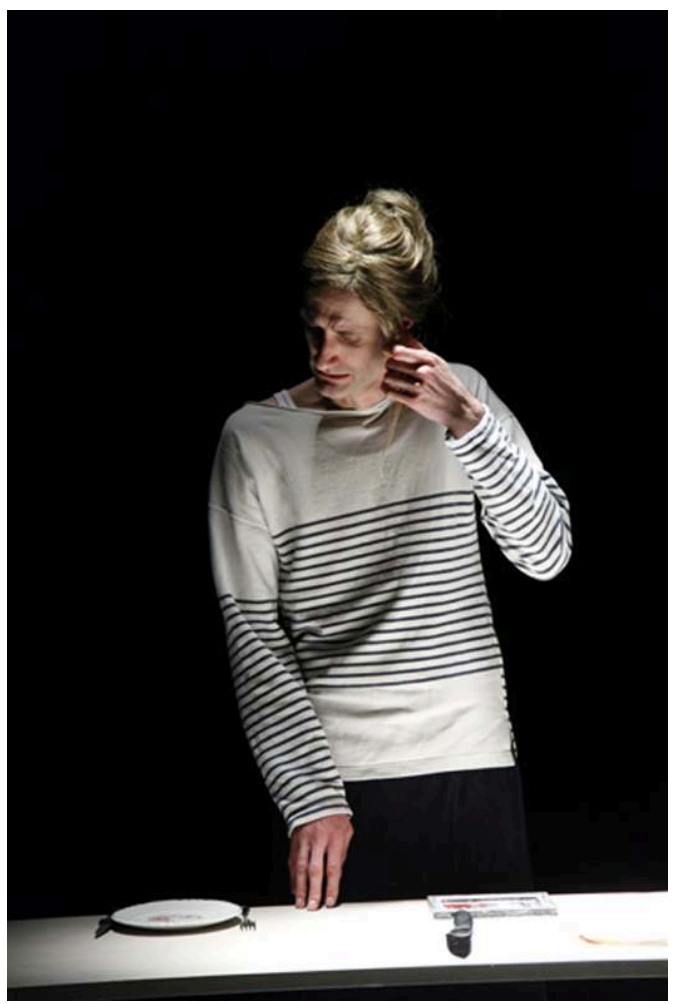

Dans Sous les Arbres (Philippe Minyana / Frédéric Maragnani). Créé au Théâtre des Abbesses en mars 2011.

Jean-Julien Kraemer

J.S. : Est-ce que, dans les écoles, les acteurs sont formés à l'entrée en scène, ou bien s'agitil d'une chose exclusivement personnelle, qui ne s'éprouve qu'au cas par cas?

G.V. : Dans les écoles, il n'y a pas d'apprentissage à l'entrée en scène ou même dans le métier ! Les différentes disciplines auxquels les acteurs sont formés (danse, sport, masque, clown etc.) permettent toutefois de développer des états toniques, des qualités d'énergies de jeu dont ils peuvent ensuite se servir. La question de l'entrée se pose de toute façon différemment pour chaque spectacle, et pas dans l'absolu.

J.S. : As-tu le souvenir d'une entrée en scène qui t'a paru particulièrement ardue?

G.V. : Cela ne m'a jamais paru normal, à moi, d'entrer en scène. La facilité serait de chercher une forme de fausse aisance, et de gaspiller de l'énergie à montrer qu'on est à l'aise et que ça va bien! Alors que la scène est un truc bizarre. C'est bizarre de rester devant des gens assis et silencieux ! C'est pourquoi je préfère essayer d'assumer l'incongruité de la situation. 
Récemment, dans Sous les arbres ${ }^{1}$ (2011), un début de scène m'a causé du souci : celui où j'interprète Linda. Il y a un in et un off dans ce début de scène : j'apparais en Linda au moment où le personnage de Tata m'interpelle («Salut Linda »), mais en même temps, je dois pousser une table et l'installer pour que la scène commence. L'attention que je mettais à prendre en charge ce changement de décor (qui devait aller vite) me donnait l'impression de démarrer à retardement la fiction : c'est un décalage que j'ai eu du mal à gérer.

J.S. : Pour le spectateur, je t'assure que ce in/off ne posait absolument aucun problème! Ce n'était qu'un jeu de convention parmi d'autres - en commençant par le fait que tu interprètes une femme, après avoir successivement été un membre du chœur, un grand type maigre, un hêtre...

G.V.: Je sais que, pour le spectateur, le moment où j'apparais en Linda (en jupe et avec une perruque blonde sur la tête) suffit à créer du jeu. C'est l'entrée claire d'un personnage nouveau, que ce personnage soit en train d'installer une table ou pas. Et ça a fini par me suffire, à moi aussi. Mais je peux tout à fait m'interroger pendant des jours sur des choses qui paraissent évidentes!

Cette entrée en scène du personnage de Linda démarrait déjà une action théâtrale (placer la table), que j'avais du mal à rattacher à la fiction du texte. J'étais préoccupé par le rythme de la scène : j'avais peur que l'énergie que je mettais à déplacer la table me fasse rater l'entrée dans le texte, qui devait être tout aussi vive, et qui, pour moi, correspondait à la véritable entrée dans la situation. Cela ne me paraissait pas juste de vraiment jouer Linda avant le démarrage du texte... Bref, je me posais des questions trop compliquées que j'ai mis du temps à dépasser.

J.S. : Est-ce que ce sentiment de décalage, d'équilibre instable entre acteur et personnage, n'est pas le propre de tous les spectacles où le jeu se fait «à vue », comme on peut le dire dans les théâtres de marionnettes ${ }^{2}$ ? Ces dernières années, tu as joué dans plusieurs pièces où non seulement tu passes d'une identité à une autre tout au long du spectacle, mais encore, où tu ne fais plus forcément d'entrées et de sorties pour signifier les différents changements de personnage que tu as à assumer. La question que je me pose est de savoir comment tu négocies l'entrée en jeu d'une nouvelle identité quand, précisément, tu es déjà entré en scène ?

G.V.: Une nouvelle identité peut se fabriquer de plein de façons différentes: en la nommant simplement ou avec l'aide d'un accessoire, un costume, etc. Si je suis déjà sur scène, tout le monde devient complice de cette fabrication. L'entrée n'est plus seulement une apparition, elle est aussi le passage visible d'un personnage à un autre, d'une fiction à une autre.

La seule chose dont je suis sûr et que je peux travailler avant les représentations est le texte que j'ai à dire. Je retravaille souvent le début des textes - celui qui ouvre le spectacle, ou ceux des différentes figures que je vais traverser: cela me donne un horizon, une sorte de goût de là où il faudrait que je sois. Et quand arrive le moment de la rencontre avec le public, il faut sans cesse se réajuster en fonction de ce qu'on sent de son énergie. 
Gaëtan Vourc'h et Laurent Charpentier

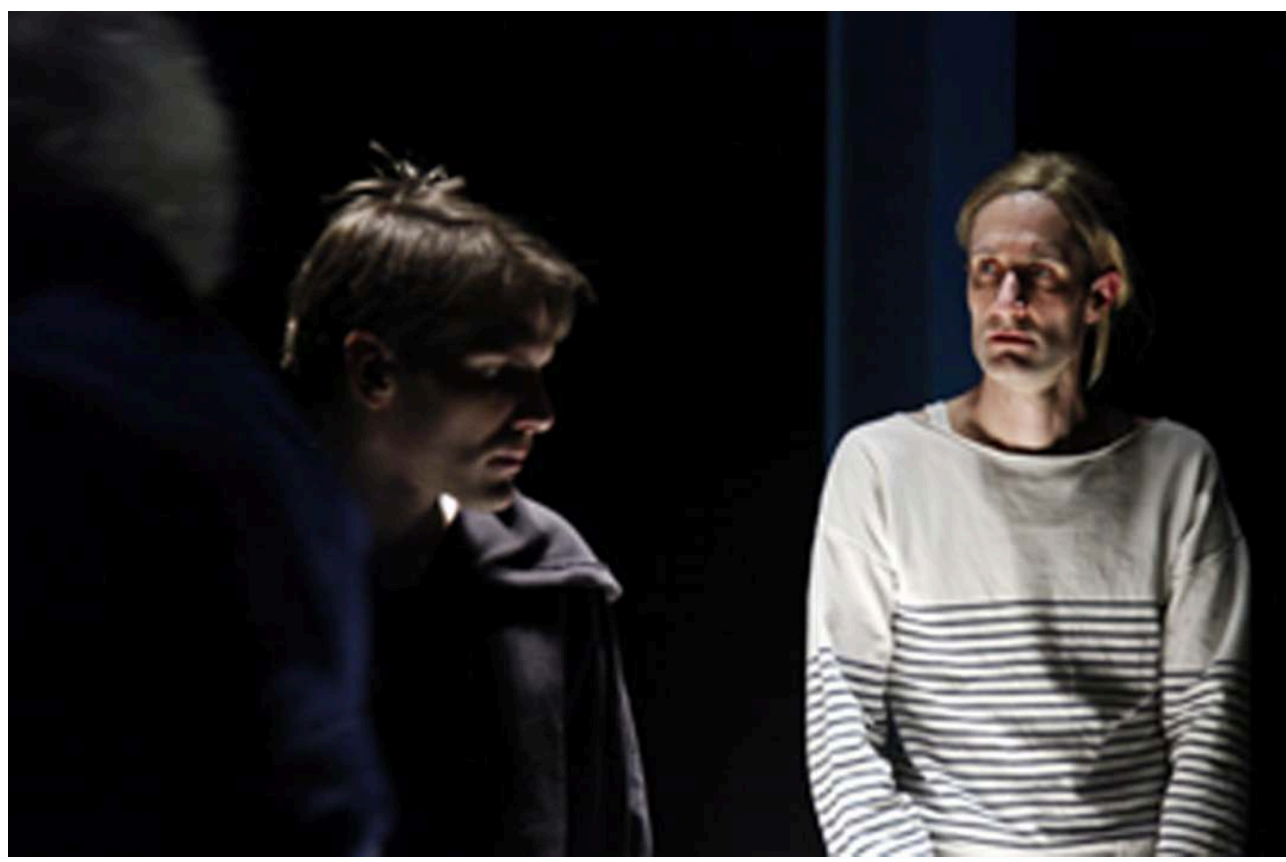

Dans Sous les Arbres (Philippe Minyana / Frédéric Maragnani). Créé au Théâtre des Abbesses en mars 2011.

Jean-Julien Kraemer

\section{J.S. : L'entrée n'est donc jamais pour toi un passage de seuil définitif?}

G.V. : Au fond, l'entrée en scène a pour moi beaucoup à voir avec ce que Stanislavski dit des « cercles d'attention » : avant de penser à l'effet que tu aimerais produire chez le spectateur, il faut commencer par te concentrer sur le cercle intérieur, sur la ou les toute(s) petite(s) chose(s) que tu as à faire. Cette micro-pensée, qui tourne en rond, qui t'obsède d'une certaine manière, fait que tu te sens très concerné par ce qui va se passer : c'est une façon de se convoquer, finalement, d'arriver tellement rempli de quelque chose (qui peut varier selon les jours) que tu es ensuite extrêmement réceptif à toutes les informations qui peuvent te parvenir.

J.S. : Est-ce que la question de l'entrée en scène se pose différemment, en tout cas, avec une acuité différente, selon que tu es le premier à entrer en scène ou que tu entres alors que le spectacle a déjà commencé ?

G.V. : Oui. Enfin, les questions « intérieures » sont les mêmes (comment se convoquer, comment s'organiser pour être attentif, etc. ...), mais la charge que tu portes sur les épaules vis-à-vis du public est moindre. Cela dit, il est finalement rare d'entrer sur une scène absolument vide, de marquer absolument le début du spectacle: la scénographie, la lumière, la musique, sont déjà des éléments d'appui pour les spectateurs, avant même que les acteurs fassent leur entrée. 


\section{NOTES}

1. Texte de Philippe Minyana (Les rêves de Margaret / Sous les arbres / De l'amour, Paris, L'Arche, 2011), mis en scène par Frédéric Maragnani au Théâtre des Abbesses en mars 2011, avec Luc Cerutti, Laurent Charpentier, Jean-Paul Dias, Bruno Galibert, Marion Lécrivain, Océane Mozas, et Gaëtan Vourc'h.

2. Voir, dans le présent dossier, "Entrée en scène des marionnettes, entrer en scène avec des marionettes. Entretien avec Sylvie Baillon, Alain Recoing et Eloi Recoing, réalisé par Julie Sermon (mai 2012)"

INDEX

Mots-clés : acteur, école 